\title{
Laser Surface Scanning Analysis in Reconstructive Rhytidectomy
}

\author{
Reto Wettstein, M.D., ${ }^{1}$ Daniel F. Kalbermatten, M.D., F.M.H., E.B.O.P.R.A.S., ${ }^{1}$ Ulrich M. Rieger, M.D., ${ }^{1}$ \\ Ralf Schumacher, ${ }^{2}$ Pavel Dagorov, M.Eng., ${ }^{2}$ and Gerhard Pierer, M.D. ${ }^{1}$ \\ ${ }^{1}$ Department of Plastic, Reconstructive, and Aesthetic Surgery, University Hospital of Basel, Spitalstrasse 21, CH-4031 Basel, \\ Switzerland \\ ${ }^{2}$ Department of Life Sciences, University of Applied Sciences of North-Western Switzerland, Gründenstrasse 40, CH-4132 \\ Muttenz, Switzerland
}

\begin{abstract}
The implementation of laser surface scanning to assess facial symmetry after unilateral face-lift procedures used to reconstruct defects after skin tumor resection is presented. Six patients who had undergone defect reconstruction with a flap raised from the subcutaneous plane were included in the study. Immediate postoperative photographic evaluation confirmed facial asymmetry because of unilateral skin tension. After a minimum follow-up period of 1 year, photographic and laser surface scanning analysis showed restored facial symmetry. In conclusion, laser surface scanning is a promising technology for objectifying results obtained and could be implemented for evaluation of the immediate and long-term effects from rhytidectomy procedures. Subcutaneous flaps without duplication or resection of the superficial musculoaponeurotic system are ideal for unilateral procedures because facial symmetry is restored after 1 year.
\end{abstract}

Key words: Facelift-Facial symmetry-3D-analysis-Skin tumor-Subcutaneous plane

Among the cardinal aims of a face-lift is the desire to reduce the number of wrinkles. Many innovative techniques and procedures have been described and continue to refine the possibilities for accomplishing this aim $[1,4,10,12]$. However, analysis of outcome after facial rejuvenation procedures remains difficult to objectify and commonly is limited to questionnaires assessing patient satisfaction and subjective evaluation of pre- and postoperative photographic material $[2,4]$.

Correspondence to Daniel F. Kalbematten,; email: daniel. kalbermatten@bluewin.ch
This study aimed to apply three-dimensional (3D) laser surface scanning for objective assessment of skin surface area after rhytidectomy procedures. Laser surface scanning is a modern technology used in mechanical and biomedical engineering that allows for precise measurement of 3D structures and surface areas $[3,5,9]$. The laser surface scanner is easy to handle, and the software necessary to process the data is commercially available.

In addition, unilateral reconstructive procedures after skin tumor eradication were chosen for the obvious advantage that every patient has an intact side of the face not subjected to surgery that serves as a control. These procedures, however, result in immediate postoperative facial asymmetry. Therefore, facial symmetry after a minimum follow-up period of 1 year was evaluated.

\section{Materials and Methods}

Patients who underwent unilateral reconstruction of the face similar to a face-lift after skin tumor resection in the temporozygomatic area were included in the current study for laser surface scanning after a minimum follow-up period of 1 year. Informed consent was obtained from all the patients.

\section{Surgical Procedure}

After tumor resection and histologic confirmation of complete tumor removal, facial integrity was reconstructed in the following manner. Flap incisions were 


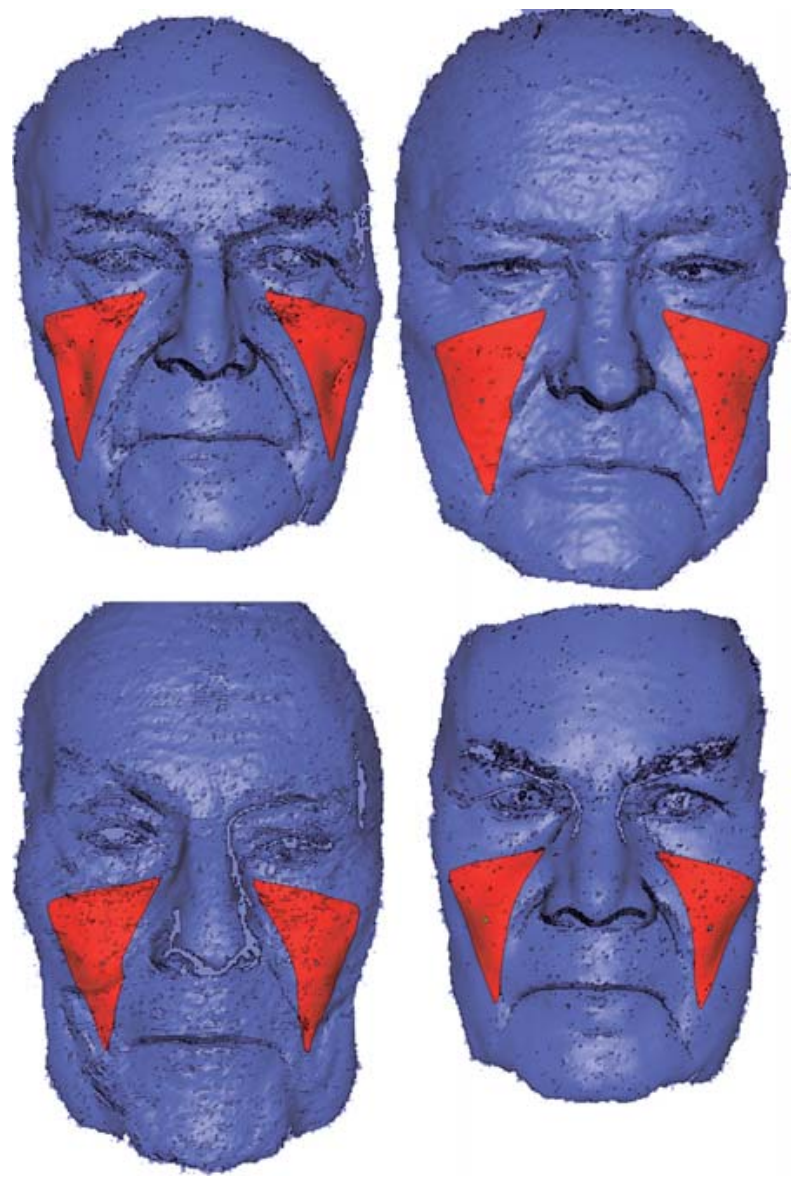

Fig. 1. Definition of the area of interest (red triangles) on the three-dimensional computer images.

made from the defect to the hairline, caudally extended along or dorsal to the hairline and into the preauricular zone, and, if necessary, continued to the retroauricular area. The flap was raised in the subcutaneous plane to the nasolabial fold anteriorly and to the mandible caudally to allow defect closure. No resection-suspension or duplication of the superficial musculo aponeurotic system (SMAS) was performed.

\section{Laser Surface Scanning}

The principle of laser surface scanning as used in the current study is that the more wrinkles present in the region of interest, the higher the surface area. After the data had been scanned and imported, regions of interest were located in each patient's cheek area where the most "lift" effect was expected (Fig. 1). This region of interest was mirrored onto the opposite side, resulting in two areas of identical size and position on the face (Fig. 2). The resulting surface areas of each region of interest were calculated and displayed in an info-dialog in square millimeters.

Technically, noncontact 3D laser surface scanning was performed with a portable device (Minolta Vivid 900 3D Digitizer; Konica Minolta Inc., Uniyoda-ku,

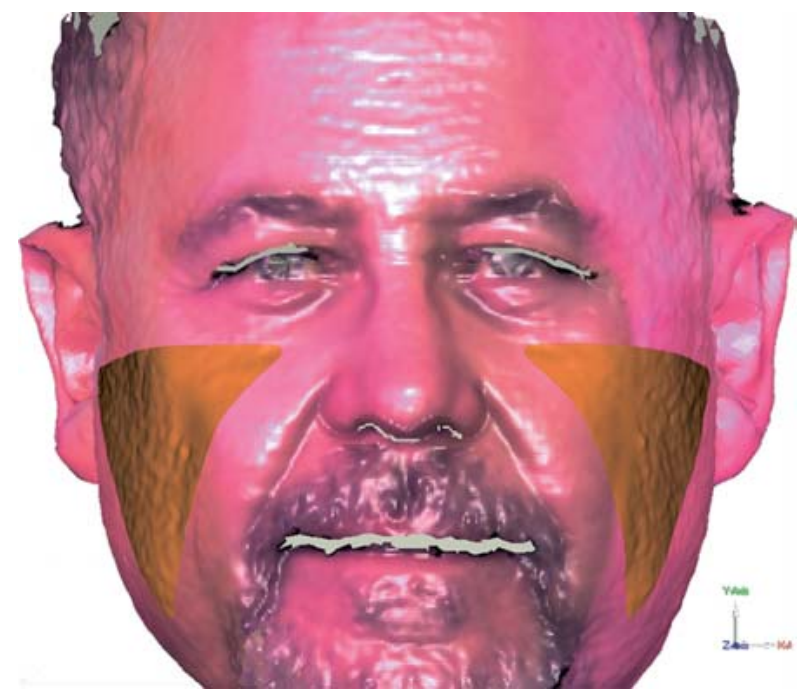

Fig. 2. Image with area of interest mirrored on the contralateral side of the face resulting in two areas of identical size.

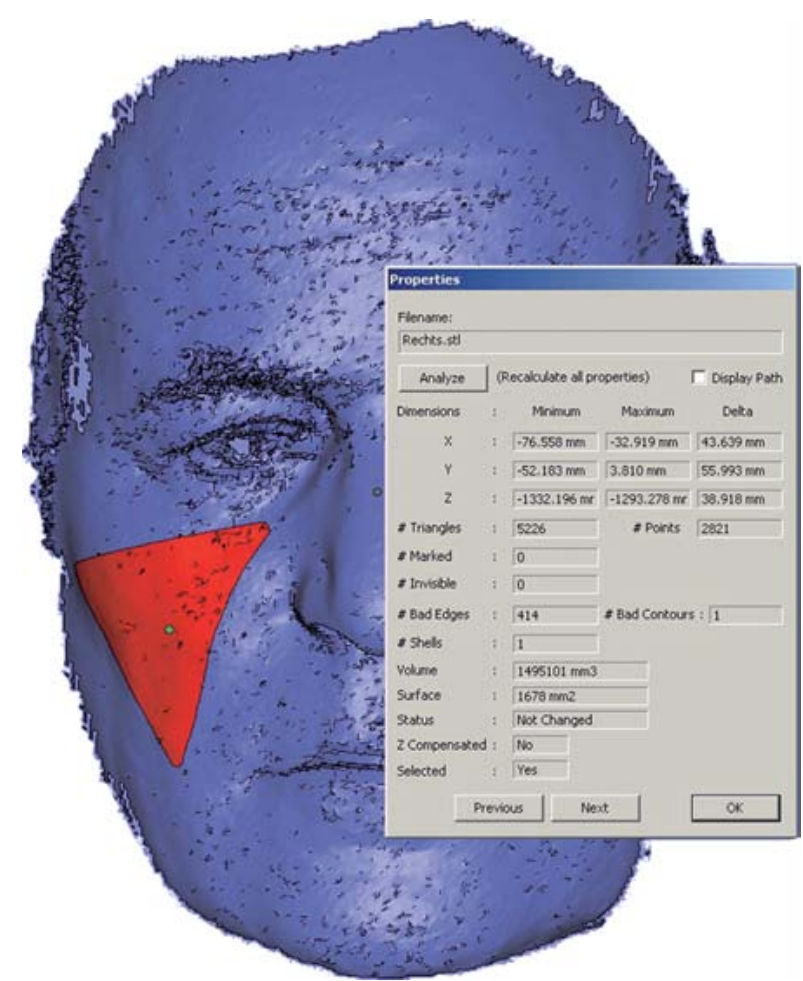

Fig. 3. Computer image with info dialogue indicating the surface area in square millimeters.

Tokyo). A 3D digitizer is an instrument that scans an object with a laser light and sends the 3D data obtained to a computer for processing to create a model of the face. The scanning process lasts less than $1 \mathrm{~s}$. The Geomagic Studio 8.0 program (Geomagic Inc., Research Triangle Park, Raleigh, North Carolina, USA) was used to import the scans. With rapid prototyping techniques (Magics RP 9.9; Materialise, 

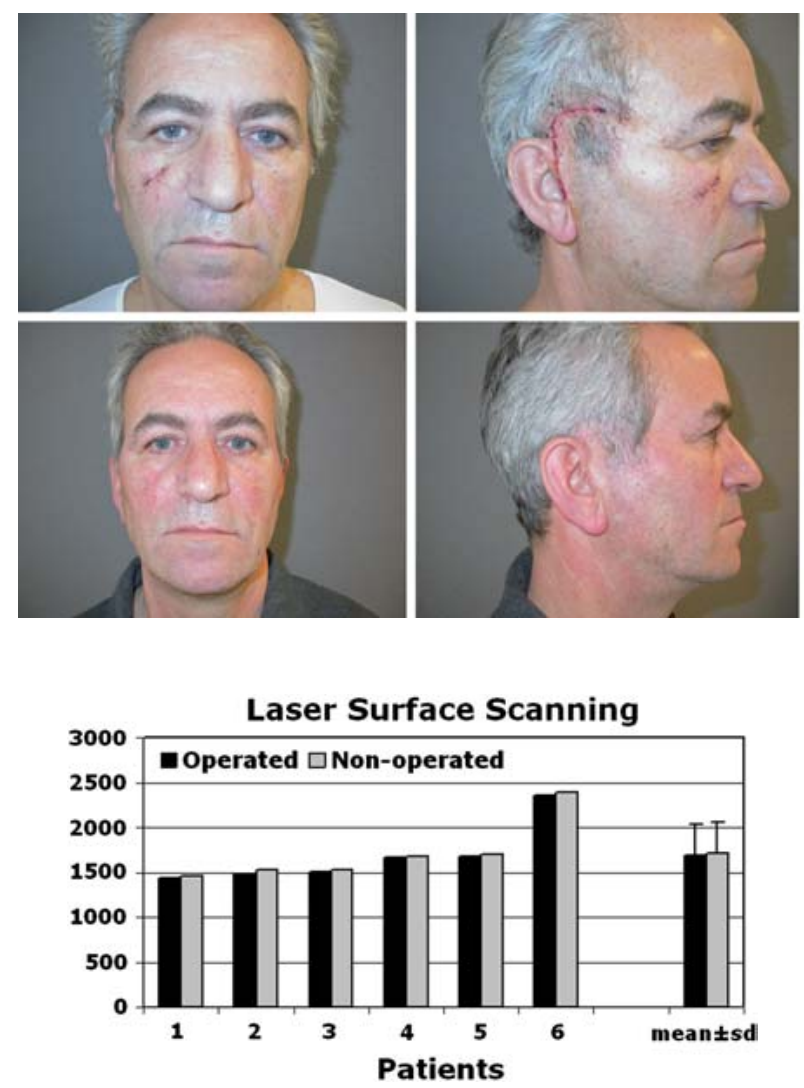

Fig. 5. Laser surface scanning results for the side of the face subjected to surgery and the side that had no surgery in square millimeters for the individual patients and the average difference. No significant difference was measured after a minimum follow-up period of 13 months.

Leuven, Belgium), polygonal punch cuboids were created and aligned on the digitalized image of the face. Using a Boolean operation, the surface areas of the cuboids were calculated and displayed in an info box (Fig. 3).

\section{Statistical Analysis}

Statistical analysis was performed with the Instat 3 program (GraphPad Software Inc., San Diego, CA). The Mann-Whitney test was used to compare surface areas in square millimeters on both the side subjected to surgery and the side that had no surgery. A $p$ value less than 0.05 was considered significant.

\section{Results}

\section{Demographic Data}

Six patients who underwent the aforementioned procedure during the period from July 2003 to December 2004 were included in the study. Their mean age was 75 years (range, $48-96$ years). Four

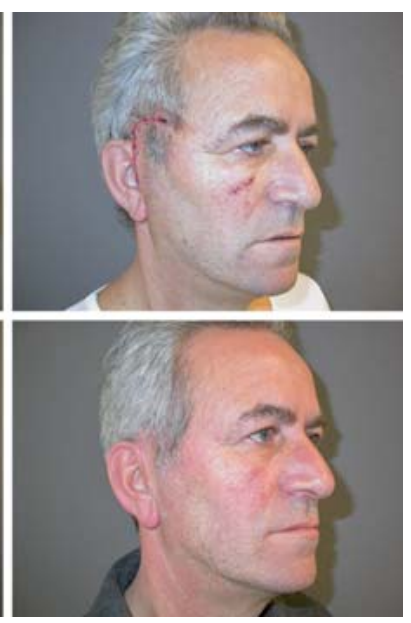

Fig. 4. Typical finding after suture material removal: unilateral rejuvenation effect resulting in facial asymmetry. Bottom-line pictures after 12 months showing relaxed position again.

patients presented with basal cell carcinoma and two patients with spinal cell carcinoma. The average follow-up period and laser surface scanning was 17 months (range 13-21 months) after surgery.

\section{Tumor Resection and Complications}

All the patients had complete tumor removal before reconstruction and no signs of recurrence at follow-up evaluation. No revisional surgeries were performed, and all flaps healed uneventfully by primary intention.

\section{Photographic Evaluation}

Immediate postoperative evaluation showed a rejuvenation effect on the side subjected to surgery, resulting in overall facial asymmetry (Fig. 4). This effect faded over time and was not noticeable after 1 year.

\section{Laser Surface Scanning}

After a minimum follow-up period of 13 months, the average surface area was $1,690 \pm 342 \mathrm{~mm}^{2}$ in the region of interest on the side of the face subjected to surgery and $1,716 \pm 348 \mathrm{~mm}^{2}$ on the side that had no surgery (Fig. 5) (nonsignificant difference). This resulted in a facial symmetry of $98 \% \pm 1 \%$.

\section{Discussion}

Laser surface scanning was used to assess skin surface area in rhytidectomy procedures. Objective measurements of the number of wrinkles in the area of interest (i.e., the cheek and malar region) was obtained for our patients. The results were consistent because all the patients had slightly fewer wrinkles on the side subjected to surgery. However, no significant differences after a mean follow-up period of 17 
months were observed. This is rather surprising because intraoperative tension after flap mobilization and defect closure demonstrates a rejuvenation effect on the side subjected to surgery.

The results obtained in this series of patients suggest that face-lift procedures in the subcutaneous plane without any intervention on the SMAS or deeper layer are ideal for unilateral reconstructive procedures because facial balance is restored to almost $100 \%$ after 1 year, which also confirms the capacity of the skin to expand.

Because all the vectors used in the current study were in the face-lift plane, future evaluation with laser scanning could help to assess the outcome objectively after different rhytidectomy procedures. Pre- and postoperative evaluation could be used to define the immediate effect and the sustainability of the different procedures in the location of the anticipated benefit. In addition, the technology used in the current study also could be used for volumetric analysis of facial contour corrections, in both aesthetic and reconstructive contexts [13].

The scanner is mobile and easy to handle. The laser surface scanning procedure is comparable with taking a Polaroid photograph. Because it lasts less than $1 \mathrm{~s}$, no distortions of the images are observed. The accuracy of the method has been validated previously in various reconstructive settings $[6-8,11]$.

In summary, laser surface scanning is reproducible and leads to a consistent assessment of skin surface area. The scanner is portable and easy to use. The subcutaneous face-lift procedures performed in the current study to reconstruct defects after tumor excision do not have a lasting rejuvenation effect, and are therefore ideal for unilateral defect reconstruction that should not affect facial symmetry. Laser surface scanning is a promising technology for objectifying results obtained, and could be implemented for evaluation of immediate and long-term effects from rhytidectomy procedures.

Acknowledgments. The authors thank Stefan de Maddalena, University of Basel, for photographic work.

\section{References}

1. Besins, T: The "R.A.R.E." technique (reverse and repositioning effect): The renaissance of the aging face and neck. Aesth Plast Surg 28:127-142, 2004
2. de la Torre, JI, Rosenberg, LZ, De Cordier, BC, Gardner, PM, Fix, RJ, Vasconez, LO: Clinical analysis of malar fat pad reelevation. Ann Plast Surg 50:244-248, 2003

3. Hill, DL, Berg, DC, Raso, VJ, Lou, E, Durdle, NG, Mahood, JK, Moreau, MJ: Evaluation of a laser scanner for surface topography. Stud Health Technol Inform 88:90-94, 2002

4. Ivy, EJ, Lorenc, ZP, Aston, SJ: Is there a difference? A prospective study comparing lateral and standard SMAS face-lifts with extended SMAS and composite rhytidectomies. Plast Reconstr Surg 98:1135-1143, 1996

5. Kalbermatten, DF, Haug, M, Schaefer, DJ, et al.: Computer-aided designed neoclavicle out of osteotomized free fibula: case report. $\mathrm{Br} J$ Plast Surg 57:668-672, 2004

6. Kovacs, L, Yassouridis, A, Zimmermann, A, Brockmann, G, Wohnl, A, Blaschke, M, Eder, M, Schwenzer-Zimmerer, K, Rosenberg, R, Papadopulos, NA, Biemer, E: Optimization of three-dimensional imaging of the breast region with three-dimensional laser scanners. Ann Plast Surg 56:229-236, 2006

7. Kovacs, L, Zimmermann, A, Brockmann, G, Baurecht, H, Schwenzer-Zimmerer, K, Papadopulos, NA, Papadopoulos, MA, Sader, R, Biemer, E, Zeilhofer, HF: Accuracy and precision of the threedimensional assessment of the facial surface using a 3D laser scanner. IEEE Trans Med Imaging 25:742-754, 2006

8. Kovacs, L, Zimmermann, A, Wawrzyn, H, Schwenzer, K, Seitz, H, Tille, C, Papadopulos, NA, Sader, R, Zeilhofer, HF, Biemer, E: Computer-aided surgical reconstruction after complex facial burn injuries: Opportunities and limitations. Burns 31:85-91, 2005

9. Kusnoto, B, Evans, CA: Reliability of a 3D surface laser scanner for orthodontic applications. $\mathrm{Am} \mathrm{J} \mathrm{Or-}$ thod Dentofacial Orthop 122:342-348, 2002

10. Noone, RB: Suture suspension malarplasty with SMAS plication and modified SMASectomy: A simplified approach to mid-face-lifting. Plast Reconstr Surg 117:792-803, 2006

11. Ramieri, GA, Spada, MC, Nasi, A, Tavolaccini, A, Vezzetti, E, Tornincasa, S, Bianchi, SD, Verze, L: Reconstruction of facial morphology from laser scanned data: Part I. Reliability of the technique. Dentomaxillofac Radiol 35:158-164, 2006

12. Ramirez, OM, Maillard, GF, Musolas, A: The extended subperiosteal face-lift: A definitive soft tissue remodeling for facial rejuvenation. Plast Reconstr Surg 88:227-236, 1991

13. Smith, DM, Aston, SJ, Cutting, CB, Oliker, A: Applications of virtual reality in aesthetic surgery. Plast Reconstr Surg 116:898-904, 2005 